\title{
Fatty liver and obesity: phenotypically correlated but genetically distinct traits in a mouse model of type 2 diabetes
}

\author{
M. Itoi-Babaya • H. Ikegami • T. Fujisawa $\cdot$ H. Ueda • \\ K. Nojima • N. Babaya $\cdot$ M. Kobayashi • S. Noso • \\ Y. Kawaguchi • K. Yamaji • M. Shibata • T. Ogihara
}

Received: 4 February 2007 / Accepted: 29 March 2007 / Published online: 5 June 2007

(C) Springer-Verlag 2007

\begin{abstract}
Aims/hypothesis Obesity and fatty liver are commonly associated with type 2 diabetes, but the genetic and functional bases linking fatty liver with obesity and diabetes are largely unknown. Our aim was to investigate the association of fatty liver with obesity and other diabetes-related phenotypes and to define the genetic control of obesity and fatty liver.

Materials and methods We established 306 F2 mice by crossing Nagoya-Shibata-Yasuda (NSY) mice, an animal model of type 2 diabetes, with control $\mathrm{C} 3 \mathrm{H}$ mice, and analysed their phenotypes. Whole-genome screening of F2 mice was performed to identify the loci responsible for fatty liver and obesity.

Results A strong association of fatty liver with obesity, hyperinsulinaemia and hyperglycaemia was observed in F2 mice. Using whole-genome screening in 306 F2 mice, we
\end{abstract}

Electronic supplementary material The online version of this article (doi:10.1007/s00125-007-0700-6) contains supplementary material, which is available to authorised users.

M. Itoi-Babaya $\cdot$ H. Ikegami $\cdot$ T. Fujisawa $\cdot$ H. Ueda $\cdot$ K. Nojima

N. Babaya $\cdot$ M. Kobayashi $\cdot$ S. Noso $\cdot$ Y. Kawaguchi $\cdot$ K. Yamaji $\cdot$

T. Ogihara

Department of Geriatric Medicine,

Osaka University Graduate School of Medicine,

Suita, Japan

H. Ikegami $(\bowtie) \cdot N$. Babaya $\cdot$ S. Noso

Department of Endocrinology, Metabolism and Diabetes,

Kinki University School of Medicine,

377-2 Ohno-higashi, Osaka-sayama,

Osaka 589-8511, Japan

e-mail: ikegami@med.kindai.ac.jp

M. Shibata

College of General Education, Aichi-Gakuin University,

Nishin, Japan mapped a new locus for fatty liver (Flln) on chromosome 6 (maximum logarithm of odds score [MLS] 10.0) and one for body weight (Bw1n) on chromosome 7 (MLS 5.1). Fl1n was linked to epididymal fat weight as well as fatty liver, but its effects were opposite in the two tissues in that the NSY allele increased liver fat but decreased epididymal fat, suggesting a role of Flln in partitioning of fat mass. The sequence of peroxisome proliferator-activated receptor $\gamma$ (Pparg), a candidate for Flln, showed allelic variation between NSY and $\mathrm{C} 3 \mathrm{H}$ mice.

Conclusions/interpretation These data suggest that fatty liver and obesity are phenotypically related but genetically independent. Loci homologous to Flln and Bwln are good candidate genes for susceptibility to fatty liver and obesity in humans.

Keywords Animal model · Fatty liver - Genetic analysis . Hepatic steatosis · Hyperglycaemia · Insulin resistance · Metabolic syndrome $\cdot$ Obesity $\cdot$ Peroxisome proliferatoractivated receptor gamma $\cdot$ QTL mapping

$\begin{array}{ll}\text { Abbreviations } \\ \text { Chr } & \text { chromosome } \\ \text { LOD } & \text { logarithm of odds } \\ \text { MLS } & \text { maximum LOD score } \\ \text { NSY } & \text { Nagoya-Shibata-Yasuda } \\ \text { PPAR } \gamma & \text { peroxisome proliferator-activated receptor } \gamma \\ \text { QTL } & \text { quantitative trait locus }\end{array}$

Introduction

Type 2 diabetes mellitus is a complex, multifactorial disease. Whereas hyperglycaemia is an essential feature of 
diabetes mellitus, obesity and insulin resistance are often associated with type 2 diabetes. In addition, fatty liver is also commonly associated with type 2 diabetes [1-3]. Increasing evidence has suggested that fatty liver is associated with obesity, insulin resistance and hyperinsulinaemia [4-6]. Despite the frequent association of fatty liver with obesity, insulin resistance and diabetes mellitus, which are common characteristics of the metabolic syndrome, the genetic basis and functional mechanisms linking fatty liver with other components of the metabolic syndrome are largely unknown. Since type 2 diabetes in humans is a heterogeneous disorder, studies using genetically and phenotypically homogeneous animal models of diabetes are necessary to elucidate the mechanisms of this disease $[7,8]$. Animal models of fatty liver, as observed in the human metabolic syndrome, however, are limited.

The Nagoya-Shibata-Yasuda (NSY) mouse strain is an inbred animal model of spontaneous type 2 diabetes [9-11]. The clinical characteristics of NSY mice resemble common forms of type 2 diabetes in humans [12], with an agedependent onset, mild obesity, insulin resistance, and impaired insulin secretion in response to glucose [12-14]. In addition, NSY mice spontaneously develop fatty liver in an age-dependent manner [15]. Type 2 diabetes in the NSY mouse is under polygenic control, with three major quantitative trait loci (QTLs) (Nidd1n, Nidd2n, Nidd3n) on mouse chromosome (Chr) 11, Chr14 and Chr6, respectively, contributing to hyperglycaemia and diabetesrelated phenotypes [16]. The genetic loci responsible for fatty liver and obesity, however, have not yet been identified. In this study, we investigated the association of fatty liver with other diabetes-related phenotypes in the F2 cross, and performed whole-genome screening to map loci responsible for fatty liver and obesity.

\section{Materials and methods}

Animals NSY [12] and C3H/He (originally purchased from Charles River Laboratories, Kanagawa, Japan) mice were maintained in the animal facilities of Osaka University Graduate School of Medicine. F2 mice were produced by crossing male NSY mice with female $\mathrm{C} 3 \mathrm{H} / \mathrm{He}$ mice, as previously reported [16]. This study conformed to the guidelines for the care and use of laboratory animals of Osaka University Graduate School of Medicine.

Study design Body weight was measured every 4 weeks from 4 to 52 weeks of age. Glucose tolerance was studied in 306 male F2 mice, using an intraperitoneal glucose tolerance test ( $2 \mathrm{~g}$ glucose $/ \mathrm{kg}$ body weight) after an overnight fast, at 12, 24, 36 and 48 weeks of age. The area under the glucose concentration curve was calculated according to the trapezoid rule. Blood glucose level was measured directly by the glucose oxidase method using Glutest E (Kyoto Daiichi Kagaku, Kyoto, Japan). Plasma insulin level was measured by RIA, as described previously [12].

All the male F2 mice were dissected under anaesthesia by intraperitoneal administration of pentobarbital at 52 weeks of age, and the liver and epididymal fat pads were immediately removed and fixed in formalin. At this time, the liver was inspected to assess the degree of fatty liver, and classified into four macroscopic grades: 1 (absent), 2 (mild), 3 (moderate) and 4 (severe). To confirm that the macroscopic grade of fatty liver assigned was appropriate, five samples of each macroscopic grade were randomly selected and subjected to blinded histological examination. Liver sections stained with Sudan III were examined using a fluorescence microscope with a picture analysis software program, IPLab Spectrum (Scanalytics, Rockville, MD, USA), and the proportion of the area stained with Sudan III was estimated in three fields for each sample.

Genotyping Genomic DNA, extracted from mouse liver using a standard phenol-chloroform method, was used as a template for the PCR. Genotypes were determined using $4 \%$ agarose gels or a fluorescence-based method [17]. A total of 109 microsatellite markers were used to cover the whole genome (average interval, $13.1 \mathrm{cM}$ ) [electronic supplementary material (ESM) Table 1].

Candidate gene sequencing Seven pairs of primers were designed to cover all seven exons and exon-intron junctions of the peroxisome proliferator-activated receptor $\gamma$ gene (Pparg). Genomic DNA of NSY and C3H mice was amplified by PCR with these primers, and the PCR products were purified using a QIAquick Gel Extraction Kit (Qiagen, Hilden, Germany). The refined products were subjected to direct sequencing using an ABI Prism BigDye Terminator v3.1 Cycle Sequencing Kit with an ABI 3100 sequencer (Applied Biosystems, Foster City, CA, USA).

Statistical analysis All data are presented as mean \pm SD or mean \pm SEM. The statistical significance of differences was estimated using unpaired Student's $t$ test and ANOVA, calculated with Statview software (SAS Institute, Cary, NC, USA). QTL analysis, permutation tests and analysis of pairwise interaction of QTLs were performed using Map Manager QTXb20 (http://www.mapmanager.org, last accessed in April 2007). Macroscopic grade was used as the phenotype. We calculated the threshold logarithm of odds (LOD) values for significant or suggestive linkage on the basis of the permutation test. 


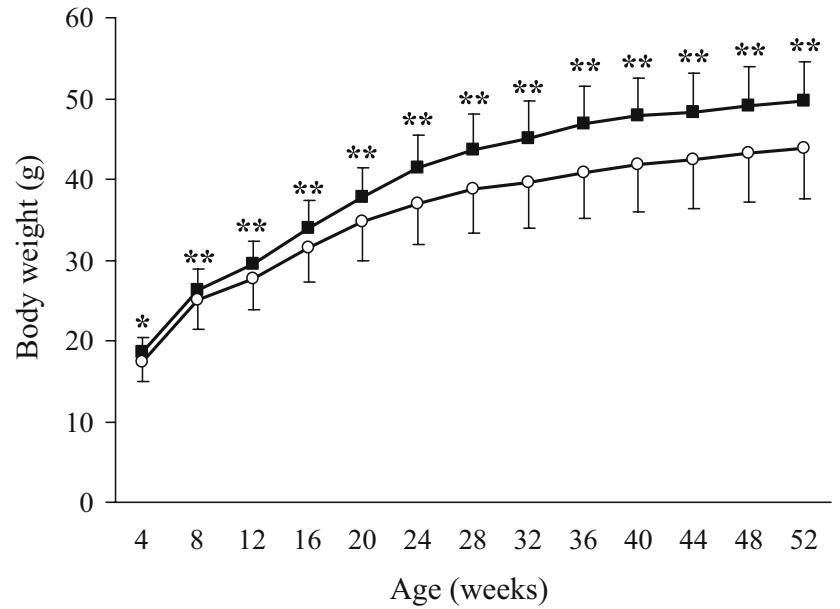

Fig. 1 Longitudinal changes in body weight in F2 mice from 4 to 52 weeks of age. Body weight of F2 mice with fatty liver $(n=130)$ was significantly greater than that of those without fatty liver $(n=176)$ at all ages. Closed squares, fatty liver (+); open circles, fatty liver (-), Data are mean \pm SD. ${ }^{*} p<0.01,{ }^{* *} p<0.0001$

\section{Results}

Association of fatty liver with obesity and diabetes-related phenotypes Figure 1 shows the longitudinal changes in body weight in F2 mice from 4 to 52 weeks of age. F2 mice with fatty liver (grade $2-4, n=130$ ) had a significantly greater body weight than those without fatty liver (grade 1, $n=176$ ) at all ages. Moreover, mice with fatty liver had higher blood glucose levels after fasting $(p<0.0001)$ and at all time points after glucose challenge (30, 60, 90 and $120 \mathrm{~min})(p<0.0001$ for all components), and a greater area under the glucose level curve $(p<0.0001$; Table 1). Plasma insulin level in mice with fatty liver was 2.5 times higher than that in those without fatty liver $(p<0.0001)$. These data indicate that fatty liver is phenotypically well correlated with diabetes-related phenotypes, i.e. obesity, insulin resistance and hyperglycaemia. The only exception was epididymal fat pad weight. Despite the strong association of fatty liver with abnormalities in all metabolic phenotypes described above (Table 1), epididymal fat pad weight was not greater, but was significantly lower in F2 mice with fatty liver than in those without fatty liver $(p<0.0005)$.

Mapping of QTLs for body weight Whole-genome mapping of 306 F2 mice identified three QTLs linked to body weight on Chr7, Chr10 and Chr15 (Fig. 2). A locus on Chr7 exhibited significant linkage with body weight, with MLS of 5.1 at 12 weeks of age (Fig. 2a), and the locus was designated $B w 1 n$ (body weight 1 in NSY). Bw1n accounted for $7 \%$ of the phenotypic trait variance. Linkage was observed in the mid-portion of $\mathrm{Chr} 7$ between the markers D7Mit81 and D7Mit238, with a wide peak. Despite the significant linkage with body weight, $B w 1 n$ on Chr7 was not linked to blood glucose or plasma insulin level. Suggestive evidence of linkage was observed for Chr10 and Chr15. Linkage was shown on Chr10, near the marker D10Mit164 (MLS, 3.4 at 12 weeks of age; Fig. 2b), and on Chr15 near the marker D15Mit113 (MLS, 2.8 at 24 weeks of age; Fig. 2c). Permutation testing showed that the threshold LOD values for suggestive QTLs for body weight were 2.1 for all age points studied, and those for significant QTLs were 3.5, 3.5, 3.4 and 3.5 at 12, 24, 36 and 48 weeks of age, respectively, based on 1,000 permutations. Analyses of pairwise interactions among the loci of 109 microsatellite markers showed no interactions among the QTLs for body weight (data not shown).

The three QTLs mapped in the NSY mouse in the present study were similar in position to some of the QTLs for body weight and adiposity previously mapped in other crosses (ESM Table 2) [18]. Among these, the central portion of Chr7 is of particular interest in that QTLs associated with body weight and adiposity have previously been reported in this region in several studies using different mouse crosses [19-21].
Table 1 Phenotypic characteristics of F2 mice with and without fatty liver at 48 weeks of age

Data are mean \pm SEM. ip GTT Intraperitoneal glucose tolerance test, $F B G$ fasting blood glucose, $B G$ blood glucose

\begin{tabular}{|c|c|c|c|}
\hline & Without fatty liver & With fatty liver & $p$ value \\
\hline$N$ & 176 & 130 & \\
\hline Body weight (g) & $43.8 \pm 0.4$ & $49.6 \pm 0.4$ & $<0.0001$ \\
\hline $\begin{array}{l}\text { BMI }\left(\mathrm{g} / \mathrm{cm}^{2}\right) \\
\text { ipGTT }\end{array}$ & $0.35 \pm 0.003$ & $0.39 \pm 0.003$ & $<0.0001$ \\
\hline $0 \min (\mathrm{FBG})(\mathrm{mmol} / \mathrm{l})$ & $4.3 \pm 0.1$ & $5.0 \pm 0.1$ & $<0.0001$ \\
\hline $30 \min \mathrm{BG}(\mathrm{mmol} / \mathrm{l})$ & $16.0 \pm 0.3$ & $18.3 \pm 0.4$ & $<0.0001$ \\
\hline $60 \min \mathrm{BG}(\mathrm{mmol} / \mathrm{l})$ & $16.4 \pm 0.4$ & $20.1 \pm 0.5$ & $<0.0001$ \\
\hline $90 \min \mathrm{BG}(\mathrm{mmol} / \mathrm{l})$ & $14.4 \pm 0.4$ & $18.4 \pm 0.6$ & $<0.0001$ \\
\hline $120 \min \mathrm{BG}(\mathrm{mmol} / \mathrm{l})$ & $11.9 \pm 0.4$ & $16.1 \pm 0.6$ & $<0.0001$ \\
\hline $\operatorname{AUC}(\mathrm{mmol} / 1 \times \mathrm{min})$ & $1644.5 \pm 38.6$ & $2024.6 \pm 47.7$ & $<0.0001$ \\
\hline Plasma insulin (pmol/1) & $1263.1 \pm 75.7$ & $3338.3 \pm 117.7$ & $<0.0001$ \\
\hline Epididymal fat pad weight (g) & $1.1 \pm 0.04$ & $0.9 \pm 0.05$ & $<0.0005$ \\
\hline
\end{tabular}




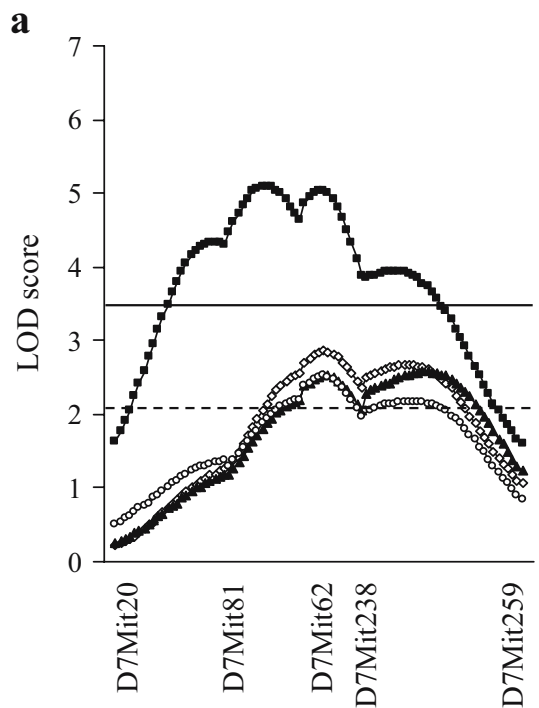

Fig. 2 LOD score curves for body weight $(n=306)$. Significant evidence of linkage (MLS, 5.1) was found in the central region of chromosome 7 between the markers D7Mit81 and D7Mit238 at 12 weeks of age (a), and the locus was designated $B w \ln$ (body weight 1 in NSY). Suggestive evidence of linkage was observed for chromosomes 10 and $15(\mathbf{b}, \mathbf{c})$. Linkage was shown on chromosome 10, near the marker D10Mit164 (MLS, 3.4) at 12 weeks of age (b),
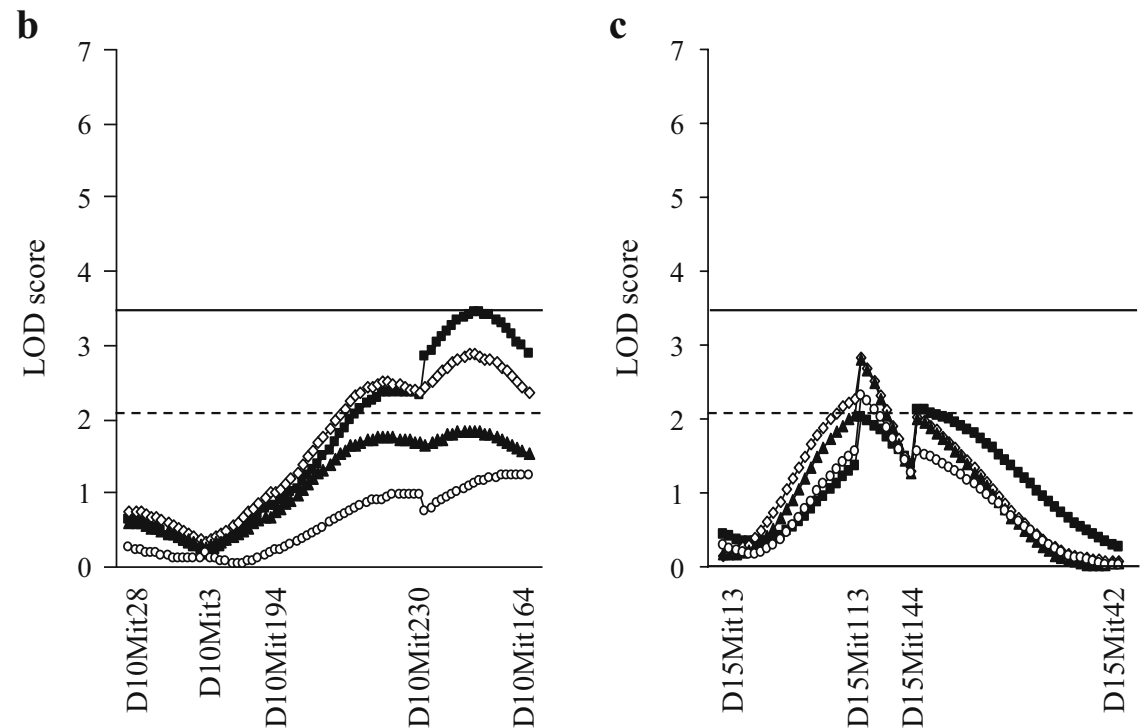

and on chromosome 15 near the marker D15Mit113 (MLS, 2.8) at 24 weeks of age (c). The horizontal dotted line indicates the threshold LOD score of 2.1 for suggestive linkage and the solid line indicates the threshold LOD score of 3.5 for significant linkage. This threshold LOD score is based on 1,000 permutations for body weight at 12 weeks of age. Closed squares, 12 weeks; open diamonds, 24 weeks; closed triangles, 36 weeks; open circles, 48 weeks

Macroscopic and microscopic findings of fatty liver Of the 306 F2 mice, fatty liver was macroscopically present in 130 $(42.5 \%)$. Of the mice with fatty liver, $13(4.2 \%)$ had grade 2 fatty liver, $98(32.0 \%)$ had grade 3, and 19 (6.2\%) had grade 4.

Microscopic examination of five liver specimens that had been macroscopically classified as grade 1 showed that hepatocytes contained only a few red spots stained with Sudan III (Fig. 3a). Semiquantitative estimation by IPLab Spectrum demonstrated that the mean area of red spots in grade 1 was $0.21 \%$ (range, $0.02-0.44 \%$ ) of the total area. Compared with these grade 1 livers, those classified as grade 2 had larger red spots (Fig. 3b), with a mean area of $1.15 \%$ (range, $0.30-1.96 \%$ ) of the total area. Similarly, grade 3
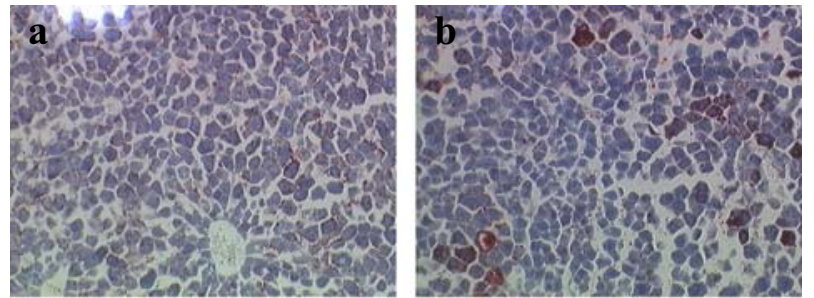

e
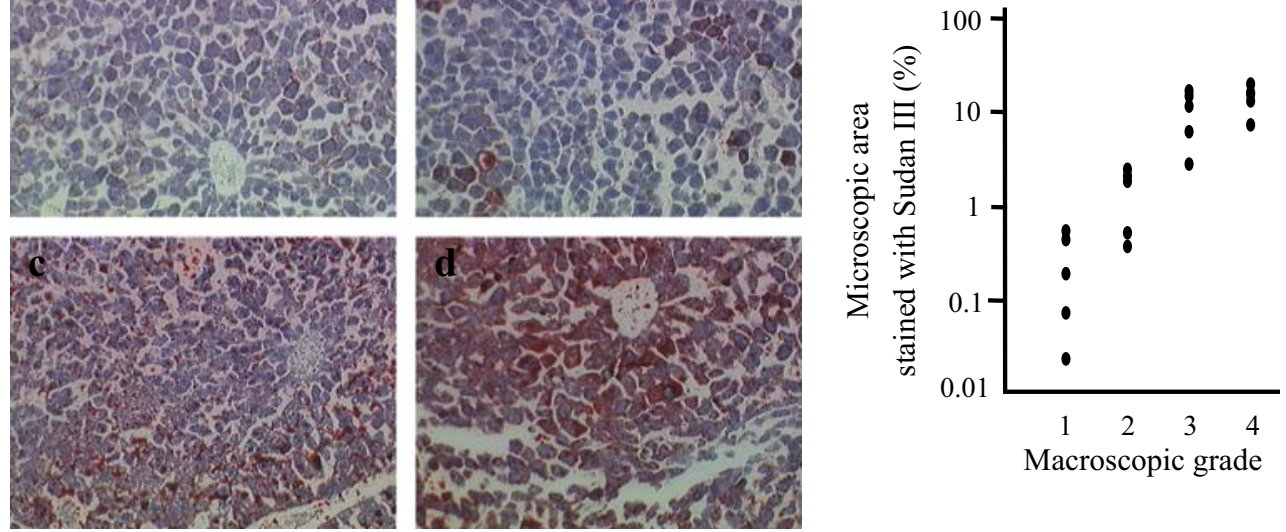

Fig. 3 a-d Histological appearance of liver in F2 mice of a cross between NSY and $\mathrm{C} 3 \mathrm{H}$. Sections from F2 mice with representative macroscopic grades were stained with Sudan III (original magnification, $\times 200$ ). Sections are representative of each macroscopic grade. a Grade 1 (fatty liver absent); b grade 2 (mild fatty liver); c grade 3 (moderate fatty liver); d grade 4 (severe fatty liver). e Relationship

between macroscopic grade and microscopic findings of fatty liver. The liver was examined with a fluorescence microscope, using the picture analysis software program IPLab Spectrum. There was a positive correlation $(r=0.80)$ between the log-transformed stained area and macroscopic grade $(n=5$ for each grade) 
livers had red vesicles that were larger (Fig. 3c) than those in grade 2, and the mean area stained was $7.95 \%$ (range, $2.2-$ $12.6 \%$ ). In liver specimens classified as grade 4 , the cytoplasm of hepatocytes was markedly occupied by fat deposits (Fig. 3d) whose area was 10.9\% (range, 5.6$15.1 \%$ ). Since these measurements of fat area were highly skewed, the log-transformed fraction of the area was investigated according to the macroscopic grade (Fig. 3e). There was a positive correlation $(r=0.80)$ between the logtransformed stained area and macroscopic grade, indicating that macroscopic grade reflects the degree of fatty liver.

QTLs for fatty liver and its linkage with other phenotypes Whole-genome screening of 306 F2 mice using the macroscopic grade of fatty liver identified highly significant linkage with fatty liver in a region on Chr6 near marker D6Mit54, with MLS of 10.0 (Fig. 4), and the locus was designated Flln (fatty liver 1 in NSY). Permutation testing showed that the threshold LOD values for suggestive and significant QTL for fatty liver were 2.1 and 3.5, respectively, based on 1,000 permutations. Flln accounted for $14 \%$ of the phenotypic trait variance. The QTL curve for fatty liver overlapped with the previously identified QTLs for epididymal fat pad weight [16]. Significant interactions of Flln were detected with the loci on Chr1, Chr3, Chr16

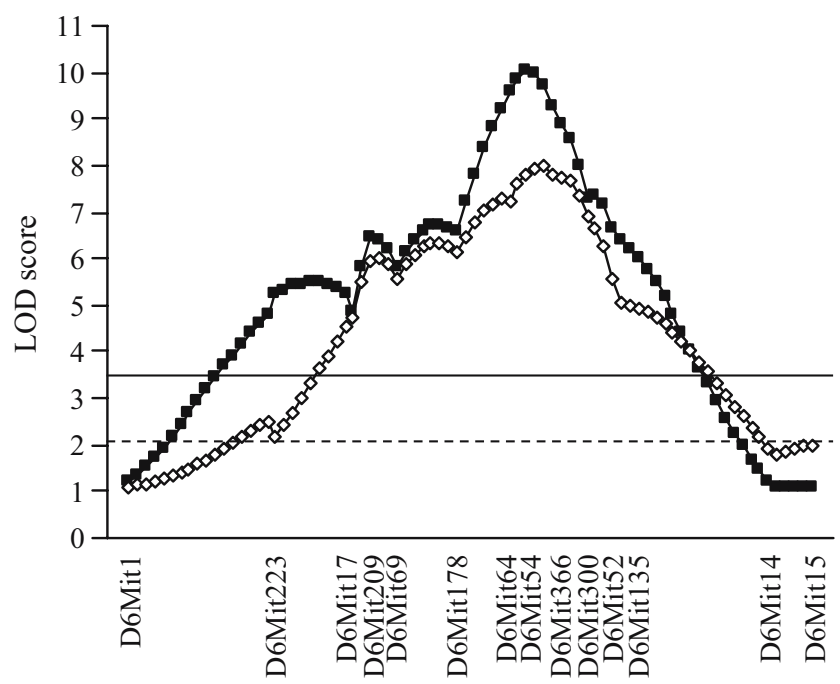

Fig. 4 LOD score curves for fatty liver score and epididymal fat pad weight $(n=306)$. Closed squares, fatty liver; open diamonds, epididymal fat pad. Significant evidence of linkage with fatty liver was found in a broad region on chromosome 6 between markers D6Mit223 (19.0 cM) and D6Mit135 (62.3 cM), with maximal LOD score of 10.0 near marker D6Mit54. This region overlapped with the previously identified QTL, Nidd3n, which showed significant evidence of linkage with epididymal fat pad weight. The horizontal dotted line indicates the threshold LOD score of 2.1 for suggestive linkage, and the solid line indicates the threshold LOD score of 3.5 for significant linkage. This threshold LOD score is based on 1,000 permutations for fatty liver and Chr17, but not with Bw1n and the loci for body weight described above (data not shown).

To clarify the effect of Flln on diabetes-related phenotypes, the macroscopic degree of fatty liver and epididymal fat pad weight were compared in 306 F2 mice relative to the genotype at Flln. F2 mice homozygous for the NSY allele at Flln marker D6Mit54 showed the greatest degree of fatty liver among the three different genotypes (Fig. 5a), while those homozygous for the $\mathrm{C} 3 \mathrm{H}$ allele displayed only a negligible degree of fatty liver. Mice heterozygous for the NSY and $\mathrm{C} 3 \mathrm{H}$ alleles showed a significantly higher degree of fatty liver than $\mathrm{C} 3 \mathrm{H}$ homozygotes, but a lower degree than did NSY homozygotes, suggesting a semidominant mode of inheritance of the NSY allele in its effect on fatty liver. For epididymal fat pad weight, in contrast, an opposite relation with these genotypes was observed (Fig. 5b). F2 mice homozygous for the NSY allele showed significantly lower epididymal fat pad weight than did $\mathrm{C} 3 \mathrm{H}$ homozygotes and heterozygotes. These data indicate that the effects of Flln on fatty liver and epididymal fat pad weight are in opposite directions, suggesting a role of Flln in the partitioning of fat mass, the NSY allele increasing fat in the liver and decreasing fat in the epididymal fat pad.

F2 mice homozygous for the NSY allele at the Flln marker locus D6Mit54 showed significantly higher plasma insulin levels than did those homozygous for the $\mathrm{C} 3 \mathrm{H}$ allele (ESM Fig. 1a), suggesting an effect of Flln not only on fatty liver but also on insulin resistance. Mice heterozygous for the NSY and $\mathrm{C} 3 \mathrm{H}$ alleles also showed significantly higher levels of plasma insulin than did $\mathrm{C} 3 \mathrm{H}$ homozygotes. No significant difference was observed in blood glucose levels among F2 mice with different genotypes at the Flln marker locus (ESM Fig. 1c). a

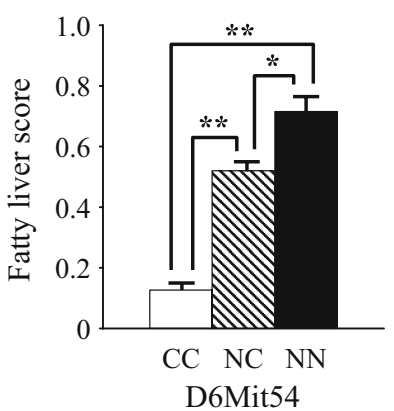

b

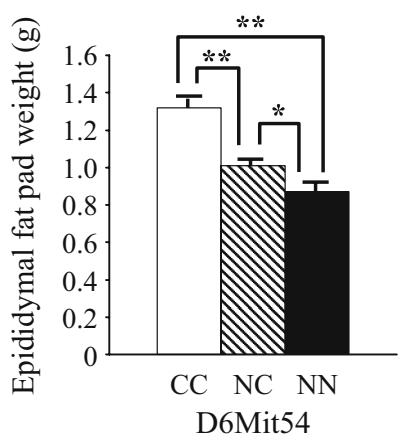

Fig. 5 a Fatty liver score in F2 mice with different genotypes at the Flln marker locus, D6Mit54. b Epididymal fat pad weight in F2 mice with different genotypes at the Flln marker locus, D6Mit54. CC, homozygous for $\mathrm{C} 3 \mathrm{H}$ allele $(n=75)$; $\mathrm{NC}$, heterozygous for NSY and $\mathrm{C} 3 \mathrm{H}$ alleles $(n=145)$; NN, homozygous for NSY allele $(n=79)$ at D6Mit54. PCR amplification failed in seven mice $(2.3 \%)$. Data are mean \pm SEM. ${ }^{*} p<0.05, * * p<0.0001$ 
Longitudinal effects of Bw1n and Fl1n on body weight No significant difference in body weight was observed in F2 mice with different genotypes at the Flln marker locus D6Mit54 at any age (Fig. 6a), indicating that the effect of Flln on fatty liver is independent of obesity. In contrast, F2 mice homozygous for the NSY allele and heterozygous for the NSY and $\mathrm{C} 3 \mathrm{H}$ alleles at the $B w 1 n$ marker locus D7Mit62 had a significantly greater body weight than mice homozygous for the $\mathrm{C} 3 \mathrm{H}$ allele, from 8 to 52 weeks of age (Fig. 6b). The data in Fig. 6 are in clear contrast to those in Fig. 1. In contrast to the clear difference in body weight in F2 mice with and without fatty liver when grouped by phenotype (Fig. 1), no difference in body weight was observed when they were grouped by genotype (Fig. 6a) despite the clear difference in the degree of fatty liver (Fig. 5a).

Pparg as a candidate for Fl1n The region on Chr6 where Flln was mapped was previously reported to be linked to diabetes-related phenotypes in other strains and this region contains several interesting candidate genes (ESM Table 3). Chromosomes homologous to the region of Flln include human $\mathrm{Chr} 3, \mathrm{Chr} 7$ and $\mathrm{Chr} 12$, and rat $\mathrm{Chr} 4$ and $\mathrm{ChrX}$.

Sequencing of Pparg as a candidate for Flln revealed 14 single-nucleotide polymorphisms; one insertion and one deletion were identified between NSY (Accession no. $\mathrm{AB} 256529$ ) and $\mathrm{C} 3 \mathrm{H}$ (accession no. AB256530) mice (Table 2). The nucleotide substitutions in the coding

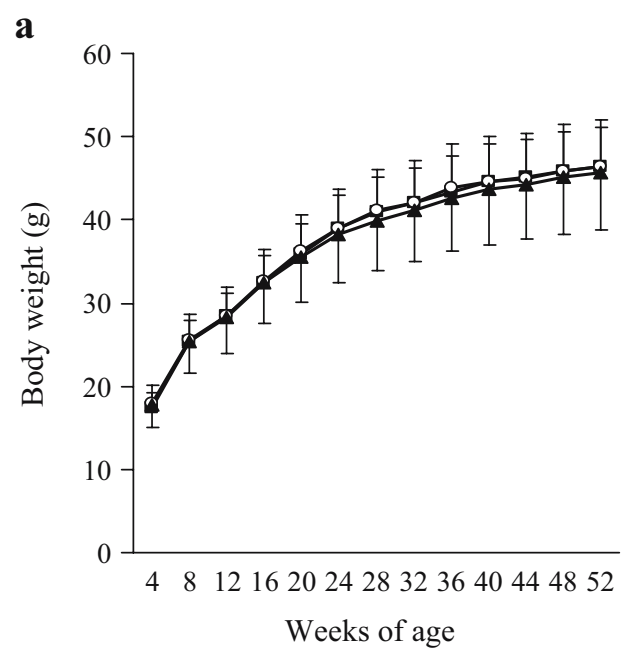

Fig. 6 Longitudinal changes in body weight in F2 mice from 4 to 52 weeks of age relative to genotypes at Flln on chromosome 6 (a) and Bw1n on chromosome 7 (b). Closed squares, N/N; open circles, $\mathrm{N} / \mathrm{C}$; closed triangles, C/C. a F2 mice homozygous for the NSY allele and heterozygous for the NSY and $\mathrm{C} 3 \mathrm{H}$ alleles at Flln marker locus D6Mit54 showed similar body weight to F2 mice homozygous for the $\mathrm{C} 3 \mathrm{H}$ allele at all ages. $\mathrm{CC}$, homozygous for $\mathrm{C} 3 \mathrm{H}$ allele $(n=75)$; $\mathrm{NC}$, heterozygous for NSY and $\mathrm{C} 3 \mathrm{H}$ alleles $(n=145)$; NN, homozygous for regions were all synonymous substitutions, and those in introns were not located at the exon-intron boundaries. When these variants were compared with reference sequences in $\mathrm{C} 57 \mathrm{BL} / 6$ mice, the sequences in NSY were identical to those in $\mathrm{C} 57 \mathrm{BL} / 6$ mice, whereas the sequences in $\mathrm{C} 3 \mathrm{H}$ were different from those in $\mathrm{C} 57 \mathrm{BL} / 6$ mice.

\section{Discussion}

In this study, F2 mice with fatty liver showed greater body weight, higher BMI and higher levels of blood glucose and plasma insulin than those without fatty liver. These data suggest a close association of fatty liver with obesity, hyperglycaemia and insulin resistance in NSY mice, as observed in humans with fatty liver.

We performed whole-genome mapping in F2 mice and identified a new susceptibility locus for fatty liver, Flln, on Chr6. The similar QTL curves for fatty liver and epididymal fat pad weight suggest that the effect of the QTL on the two phenotypes is genetically linked. In fact, significant differences in both fatty liver and epididymal fat pad weight were observed between F2 mice with different genotypes at the Flln locus (Fig. 5). To our surprise, however, the effects of the NSY allele on fatty liver and epididymal fat were in opposite directions, in that the NSY allele increased fat accumulation in the liver but decreased fat mass in the epididymal fat pad. These data suggest that Flln affects the

b

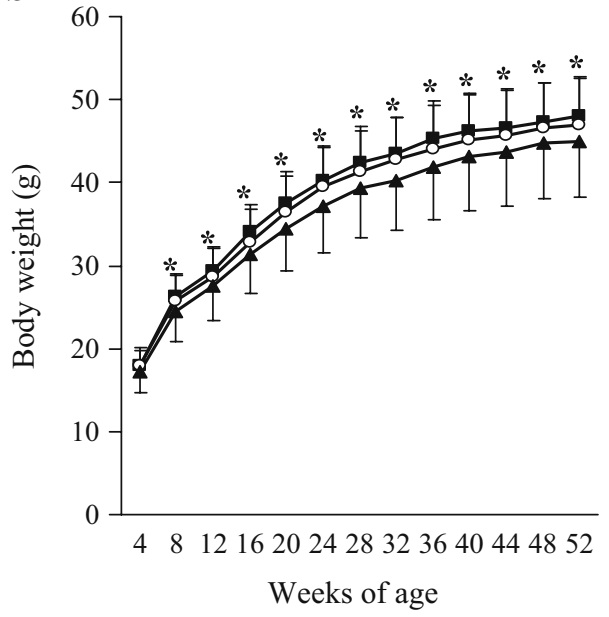

NSY allele $(n=79)$ at D6Mit54. PCR amplification failed in seven mice $(2.3 \%)$. b F2 mice homozygous for the NSY allele and heterozygous for the NSY and $\mathrm{C} 3 \mathrm{H}$ alleles at the $B w 1 n$ marker locus D7Mit62 showed markedly greater body weight than that in F2 mice homozygous for the $\mathrm{C} 3 \mathrm{H}$ allele, from 8 to 52 weeks of age. $\mathrm{CC}, n=76$; $\mathrm{NC}, n=117 ; \mathrm{NN}, n=58$ at D7Mit62. PCR amplification failed in 55 mice $(18.0 \%)$. Data are mean \pm SD. ${ }^{*} p<0.01$ for $\mathrm{NN}$ vs $\mathrm{CC}$ and $p<0.05$ for $\mathrm{NC}$ vs CC 
Table 2 Sequence analysis of Pparg

The information on reference sequences (C57BL/6J strain) is derived from a web database (http://www.ensembl.org/

Mus_musculus); NSY mice (accession no. AB256529), $\mathrm{C} 3 \mathrm{H}$ mice

(accession no. AB256530). Primer sequences for the PCR are available on request. $n t$ Nucleotide

${ }^{\mathrm{a}}$ Insertion

${ }^{\mathrm{b}}$ Deletion

\begin{tabular}{|c|c|c|c|c|}
\hline & Position & Reference & NSY & $\mathrm{C} 3 \mathrm{H}$ \\
\hline Intron 1 & (nt 267) & - & - & aaatgta $^{\mathrm{a}}$ \\
\hline Intron 2 & (nt 18010) & $\mathrm{t}$ & $\mathrm{t}$ & $\mathrm{c}$ \\
\hline Intron 2 & (nt 18026) & - & - & $\operatorname{aggattgg}^{\mathrm{b}}$ \\
\hline Intron 3 & (nt 19611) & $\mathrm{t}$ & $\mathrm{t}$ & a \\
\hline Intron 5 & (nt 41252) & $\mathrm{a}$ & $\mathrm{a}$ & $\mathrm{t}$ \\
\hline Intron 5 & (nt 41278) & $\mathrm{t}$ & $\mathrm{t}$ & $\mathrm{c}$ \\
\hline Exon 6 & (nt 50888) & $\mathrm{G}(\mathrm{glu})$ & G (glu) & A (glu) \\
\hline Exon 6 & (nt 50936) & C (ala) & $\mathrm{C}$ (ala) & $\mathrm{T}$ (ala) \\
\hline Exon 6 & (nt 50972) & $\mathrm{T}$ (pro) & $\mathrm{T}$ (pro) & $\mathrm{C}$ (pro) \\
\hline Exon 6 & (nt 50990) & $\mathrm{T}(\operatorname{asp})$ & $\mathrm{T}$ (asp) & $\mathrm{C}$ (asp) \\
\hline Exon 6 & (nt 51209) & $\mathrm{C}$ (asp) & $\mathrm{C}$ (asp) & $\mathrm{T}$ (asp) \\
\hline Exon 7 & (nt 67968) & $\mathrm{T}$ (asn) & $\mathrm{T}$ (asn) & $\mathrm{C}$ (asn) \\
\hline $3^{\prime}$ downstream & (nt 68404) & $\mathrm{t}$ & $\mathrm{t}$ & c \\
\hline $3^{\prime}$ downstream & (nt 68485) & $\mathrm{a}$ & $\mathrm{a}$ & $\mathrm{g}$ \\
\hline $3^{\prime}$ downstream & (nt 68535) & $\mathrm{t}$ & $\mathrm{t}$ & $\mathrm{c}$ \\
\hline $3^{\prime}$ downstream & (nt 68632) & $\mathrm{a}$ & $\mathrm{a}$ & $\mathrm{g}$ \\
\hline
\end{tabular}

partitioning of fat, the NSY allele causing accumulation of more fat in the liver and less fat in the epididymal fat pad. Longitudinal analysis of body weight showed that the Flln locus does not affect body weight at any age (Fig. 6a), further indicating that the effect of Flln on fatty liver is independent of obesity. This is in clear contrast to the significant difference in body weight between F2 mice with and without fatty liver when grouped phenotypically (Fig. 1).

The plasma insulin level in F2 mice with the NSY allele at the Fl1n locus was significantly higher than that in mice without the NSY allele (ESM Fig. 1a). In contrast, the $B w 1 n$ locus did not affect the plasma insulin level (ESM Fig. 1b). These data indicate that the Flln locus is more closely related to insulin resistance than the $B w 1 n$ locus, suggesting a link between fatty liver and insulin resistance. This observation is also consistent with recent reports in humans showing that a relatively modest weight reduction leads to a marked reduction of intrahepatic lipid content, and improved insulin sensitivity and glucose metabolism $[22,23]$.

Blood glucose levels were not significantly different among F2 mice with different genotypes at the Fl1n locus (ESM Fig. 1c), suggesting that, despite its effect on insulin resistance, the Flln locus alone is not sufficient to cause hyperglycaemia. In our previous genome scan, suggestive evidence of linkage with hyperglycaemia was observed on the same chromosome (Chr6) as Flln, but the QTL curve for this locus was quite different from that for Flln, with peak linkage located around $61.4 \mathrm{cM}$ more telomeric to the Flln locus [16]. This QTL and other QTLs on different chromosomes (Nidd1n and/or Nidd $2 n$ on Chr11 and Chr14 respectively) in addition to Flln may contribute to the conversion of fatty liver and insulin resistance to diabetes.
PPAR $\gamma$ has been reported to be related to the metabolic syndrome, fatty liver and type 2 diabetes in several studies $[24,25]$. Overproduction of PPAR $\gamma 2$ in the liver has recently been reported to induce fatty liver while markedly decreasing epididymal fat [26], making Pparg a good candidate gene for Flln. Sequencing of Pparg revealed several nucleotide substitutions between NSY and $\mathrm{C} 3 \mathrm{H}$ mice. Further studies are needed to clarify whether some of the substitutions identified in the present study are functional and contribute to phenotypic differences between NSY and $\mathrm{C} 3 \mathrm{H}$ mice.

Previous studies have demonstrated that genetic factors play a major role in the development of obesity [27]. We searched for loci linked to body weight and obesity and identified Bw1n on Chr7. Two QTLs on Chr10 and Chr15 also showed suggestive evidence of linkage with body weight. Candidate genes as well as the human orthologues of mouse Chr7, Chr10 and Chr15, where the QTLs for body weight were mapped, are shown in ESM Table 2. Some loci were detected in the region related to obesity in studies in humans [18-20, 28, 29].

Despite the close association of fatty liver with obesity in F2 mice as a phenotype, genetic control of fatty liver by Flln is independent of obesity, suggesting the possibility of distinguishing the impact of fatty liver on metabolic abnormalities from that of obesity by the use of this model. A definitive conclusion, however, on the relative degrees of importance of the effect of fatty liver and obesity on metabolic abnormalities will come from studies in congenic strains for Flln and Bwln. Such congenic strains are now being established; this will facilitate the genetic dissection and elucidation of the molecular mechanisms of complex interactions among fatty liver, obesity, insulin resistance and type 2 diabetes. The data also suggest that homologous 
regions in humans are strong candidates for genes predisposing to fatty liver and the associated metabolic syndrome.

Acknowledgements We thank Miss Miyuki Moritani for her skilful technical assistance. This study was supported by a Grant-in-Aid for Scientific Research on Priority Areas, a Grant-in-Aid for Exploratory Research, a Grant-in-Aid for Scientific Research from the Ministry of Education, Culture, Sport and Science and Technology of Japan, and a Grant-in-Aid from Takeda Science Foundation.

Duality of interest statement All authors declare that there is no conflict of interest concerning this study.

\section{References}

1. Stone BG, Van Thiel DH (1985) Diabetes mellitus and the liver. Semin Liver Dis 5:8-28

2. Foster KJ, Griffith AH, Dewbury K, Price CP (1980) Liver disease in patients with diabetes mellitus. Postgrad Med J 56:767-772

3. Silverman JF, Pories WJ, Caro JF (1989) Liver pathology in diabetes mellitus and morbid obesity. Clinical, pathological, and biochemical considerations. Pathol Annu 24:275-302

4. Marchesini G, Brizi M, Bianchi G et al (2001) Nonalcoholic fatty liver disease: a feature of the metabolic syndrome. Diabetes 50:1844-1850

5. Angelico F, Del Ben M, Conti R et al (2003) Non-alcoholic fatty liver syndrome: a hepatic consequence of common metabolic diseases. J Gastroenterol Hepatol 18:588-594

6. Yki-Jarvinen H, Westerbacka J (2005) The fatty liver and insulin resistance. Curr Mol Med 5:287-295

7. Ktorza A, Bernard C, Parent V et al (1997) Are animal models of diabetes relevant to the study of the genetics of non-insulindependent diabetes in humans? Diabetes Metab 23(Suppl 2):38-46

8. Moore KJ, Nagle DL (2000) Complex trait analysis in the mouse: the strengths, the limitations and the promise yet to come. Annu Rev Genet 34:653-686

9. Shibata M, Yasuda B (1980) New experimental congenital diabetic mice (NSY mice). Tohoku J Exp Med 130:139-142

10. Ueda H, Ikegami H, Shibata M, Ogihara T (2001) The NSY mouse: an animal model of human type 2 diabetes mellitus with polygenic inheritance. In: Sima A, Shafrir E (eds) Animal models of diabetes: a primer. Harwood Academic Publishers, Newark, pp 185-195

11. Beck JA, Lloyd S, Hafezparast M et al (2000) Genealogies of mouse inbred strains. Nat Genet 24:23-25

12. Ueda H, Ikegami H, Yamato E et al (1995) The NSY mouse: a new animal model of spontaneous NIDDM with moderate obesity. Diabetologia 38:503-508

13. Ueda H, Ikegami H, Kawaguchi Y et al (2000) Age-dependent changes in phenotypes and candidate gene analysis in a polygenic animal model of type II diabetes mellitus: NSY mouse. Diabetologia 43:932-938

14. Hamada $Y$, Ikegami $H$, Ueda $H$ et al (2001) Insulin secretion to glucose as well as nonglucose stimuli is impaired in spontaneously diabetic Nagoya-Shibata-Yasuda mice. Metabolism 50:1282-1285

15. Ueda H, Ikegami H, Kawaguchi Y et al (2000) Paternal-maternal effects on phenotypic characteristics in spontaneously diabetic Nagoya-Shibata-Yasuda mice. Metabolism 49:651-656

16. Ueda H, Ikegami H, Kawaguchi Y et al (1999) Genetic analysis of late-onset type 2 diabetes in a mouse model of human complex trait. Diabetes 48:1168-1174

17. Reed PW, Davies JL, Copeman JB et al (1994) Chromosomespecific microsatellite sets for fluorescence-based, semi-automated genome mapping. Nat Genet 7:390-395

18. Snyder EE, Walts B, Perusse L et al (2004) The human obesity gene map: the 2003 update. Obes Res 12:369-439

19. van Tilburg JH, Sandkuijl LA, Strengman E, Pearson PL, van Haeften TW, Wijmenga C (2003) Variance-component analysis of obesity in type 2 diabetes confirms loci on chromosomes $1 \mathrm{q}$ and 11q. Obes Res 11:1290-1294

20. Atwood LD, Heard-Costa NL, Cupples LA, Jaquish CE, Wilson PW, D'Agostino RB (2002) Genomewide linkage analysis of body mass index across 28 years of the Framingham Heart Study. Am J Hum Genet 71:1044-1050

21. Warden CH, Fisler JS, Shoemaker SM et al (1995) Identification of four chromosomal loci determining obesity in a multifactorial mouse model. J Clin Invest 95:1545-1552

22. Tiikkainen M, Bergholm R, Vehkavaara S et al (2003) Effects of identical weight loss on body composition and features of insulin resistance in obese women with high and low liver fat content. Diabetes 52:701-707

23. Petersen KF, Dufour S, Befroy D, Lehrke M, Hendler RE, Shulman GI (2005) Reversal of nonalcoholic hepatic steatosis, hepatic insulin resistance, and hyperglycemia by moderate weight reduction in patients with type 2 diabetes. Diabetes 54:603-608

24. Lehrke M, Lazar MA (2005) The many faces of PPARgamma. Cell 123:993-999

25. Jones JR, Barrick C, Kim KA et al (2005) Deletion of PPARgamma in adipose tissues of mice protects against high fat diet-induced obesity and insulin resistance. Proc Natl Acad Sci USA 102:6207-6212

26. Uno K, Katagiri H, Yamada T et al (2006) Neuronal pathway from the liver modulates energy expenditure and systemic insulin sensitivity. Science 312:1656-1659

27. Bell CG, Walley AJ, Froguel P (2005) The genetics of human obesity. Nat Rev Genet 6:221-234

28. Hanson RL, Ehm MG, Pettitt DJ et al (1998) An autosomal genomic scan for loci linked to type II diabetes mellitus and bodymass index in Pima Indians. Am J Hum Genet 63:1130-1138

29. Li WD, Dong C, Li D, Zhao H, Price RA (2004) An obesity-related locus in chromosome region 12q23-24. Diabetes 53:812-820 